Investigaciones Fenomenológicas, n. 9, 2012, 249-268.

e-ISSN: $1885-1088$

\title{
EL CONCEPTO METODOLÓGICO DE REFLEXIÓN en Husserl y en Ricoeur
}

\author{
Roberto C. F. Menéndez \\ Universidad de Granada, España / \\ Université de Rouen, Francia \\ Roberto_obarri@yahoo.es
}

\begin{abstract}
Resumen: Este trabajo describe el concepto de reflexión en la fenomenología de Edmund Husserl y en la hermenéutica de Paul Ricoeur, especialmente en sus trabajos metodológicos y de fundamentación de sus respectivas filosofías. La atención está puesta principalmente en las semejanzas y diferencias entre ambas nociones de reflexión. Con ello se pretende conducir al lector hacia la pregunta que interroga por el sentido de la actividad reflexiva, entendida como capacidad de descubrir y analizar las propias operaciones subjetivas implicadas en la constitución de la vida y del mundo. En último término se pretende revisar el alcance de ambas filosofías y la posibilidad de una conjunción entre ambas.
\end{abstract}

Palabras clave: Reflexión, fenomenología, hermenéutica, Husserl, Ricoeur.

\begin{abstract}
This essay describes the concept of reflection in Edmund Husserl's phenomenology and Paul Ricoeur's hermeneutics, especially in the methodological and foundation works of their philosophies. The issue is mainly focused on the similarities and differences between both notions of reflection. This tries to lead the reader towards wondering about the meaning of the reflective activity, being understood as the ability to discover and analyse the very subjective operations involved in the constitution of life and world. Finally, the aim is to review the scope of both philosophies and the possibility of a conjunction between them.
\end{abstract}

Keywords: Reflection, phenomenology, hermeneutic, Husserl, Ricoeur.

\section{INTRODUCCIÓN}

El asombro con el que surge la filosofía, como ciencia que investiga los diferentes objetos y regiones de la naturaleza, así como el ser en general, conduce a un segundo asombro, que es el asombro ante el sentido no evidente del mismo asombro, o dicho en otras palabras, el asombro de sentirse y saberse el sujeto un ser interrogante que, por más que quiera, no sale de su asombro. El misterio de que haya algo en vez de nada cubre al mundo de una neblina maravillosa que acaba por envolver al mismo ser humano, volviéndolo sobre sí mismo y haciéndole partícipe del enigma que se interroga. De algún modo, el primer asombro lleva al segundo, y el movimiento incesante de estas dos caras de la misma moneda que nunca deja de dar vueltas en el aire generan la ex- 
pectativa del sentido: del sentido del mundo y del sentido de la filosofía que se pregunta por el mundo $-\mathrm{y}$ que de algún modo, también se pregunta por sí misma. Esta expectativa de sentido impregna el filosofar de componentes emocionales encontrados: son la fruición y la frustración de un interrogar siempre postergado, plagado de más y más horizontes que apuntan al infinito.

La historia de la filosofía occidental, de un modo muy esquemático, se entiende a sí misma según dos modelos muy generales en cuando al sentimiento del asombro se refiere: por un lado, el asombro ante el mundo y ante lo trascendente, con la consiguiente investigación de la naturaleza y el cuestionamiento de la Trascendencia. Por otro lado, el asombro ante las propias capacidades racionales, ante el misterio de la inmanencia, y la pregunta por las condiciones subjetivas de la experiencia y el conocimiento. Una pregunta condensa con gran acierto la primera forma de investigación: "¿por qué el ser y no más bien la nada?". En cuanto a la segunda, quizá más compleja en su modo de experimentarse, podríamos preguntar, simplificando, "¿por qué hay, además del ser, una conciencia capaz de pensar que, en efecto, podría no haber nada?".

Paradigmáticamente se utiliza el ejemplo de Descartes para situar cronológicamente el comienzo de esta segunda navegación. Esta delimitación está lejos de ser rígida. De algún modo San Agustín está del segundo lado pese a remitir a la Trascendencia sus interrogaciones íntimas, y de algún modo Kant se dirige al primero pese a preguntarse por las condiciones subjetivas del conocimiento. En el tiempo de Descartes la investigación de la naturaleza pasaba por un momento de tanto éxito que los científicos, extasiados por la saturación de sentido de sus investigaciones, no tenían tiempo para preguntarse "¿qué sentido tiene conocer científicamente el mundo?". La respuesta a esta pregunta estaba presupuesta en el progreso mismo de la ciencia, que confirmaba de modo suficiente el sentido de la ciencia. A medida que las ciencias avanzan, consolidándose cada vez más sus métodos de investigación y de comunicación, surgirá tímidamente una pregunta mucho más difícil de responder, pues no puede plantearse en los mismos términos ni con los mismos métodos de la ciencia natural: "¿cuál es el sentido del conocimiento?".

En general, un cierto presentimiento del sentido de la ciencia, y de la conciencia de sus avances y sus carencias, hunde estas cuestiones $y$, con ellas, el trabajo de la filosofía, al tiempo que la investigación de la naturaleza, agarrada desde un principio a tales presentimientos, continúa perfeccionándose y espe- 
cializándose. La ciencia predica con el ejemplo y su mejor ejemplo es el progreso. Más allá no caben preguntas, sino un vago interrogar metafísico, puro diletantismo. Ahora bien, filosofías como la de Schopenhauer, alejadas de toda relación entre ciencia y conocimiento, son ejemplo de este segundo gran movimiento de la filosofía que se pregunta por el sentido de una conciencia capaz de preguntarse por su propio destino. La ciencia, volcada sobre el mundo de lo positivo y acumulando un éxito tras otro, no tiene tiempo para volverse sobre sí misma y preguntar por la "conciencia de la ciencia" y, mucho menos, de un modo más general, para ponerse a fundamentar, según nuevos métodos, una "ciencia de la conciencia", una "ciencia de la experiencia de la conciencia".

En este contexto, una filosofía hará de la pregunta por el sentido del conocimiento su tarea infinita de investigación y explicitación. La fenomenología de Husserl no cuestiona el éxito o fracaso de unas u otras ciencias, sino que se pregunta por el sentido de su actividad y por el sentido último del conocer. Para ello, todas las precisiones metodológicas de esta nueva filosofía no están enfocadas directamente a perfeccionar el conocimiento del mundo, sino a precisar el sentido mismo de ese conocimiento en la misma experiencia entendida como actividad, como actividad humana. Con tal finalidad, la fenomenología elabora una metodología compleja y rica que apunta a la actividad que quedaba impensada en la investigación científica de la naturaleza. Con ello, convierte la pregunta por el sentido del conocimiento en una metodología destinada a fundamentar la filosofía como ciencia, aquella que sirve para plantear el sentido del conocimiento en general, englobando con ello el sentido de todas las ciencias disciplinares. Después, otros filósofos, impresionados ante el detalle y la profundidad de la fenomenología, comenzarán a vislumbrar la posibilidad de aplicar el nuevo método a un ámbito más amplio, menos llamado a preguntar por el sentido último del conocimiento, y más a interrogar por el sentido de la experiencia en general, por el origen subjetivo del mundo y de la vida.

En este texto trataremos de comprender cómo la reflexión, como concepto metodológico de la fenomenología, y haciendo de bisagra entre la atención al mundo y la vuelta sobre sí, puede ponernos ante la pista de la interrogación que va del asombro ante el mundo al asombro ante la conciencia del asombro, es decir, no sólo ante la conciencia del mundo sino también ante la conciencia 
de sí -lo que Husserl llamará el "maravilloso ser para sí mismo del ego"1. Los actos reflexivos serán entendidos, de nuevo, como las bisagras del gran gozne de la reducción trascendental, que articula el movimiento que va del correlato de la conciencia intencional a la subjetividad trascendental donde opera la actividad significativa del mundo. En segundo lugar, trataremos de ver hasta qué punto el concepto de reflexión en Paul Ricoeur sirve para articular metodológicamente su hermenéutica, conjugando de igual modo una subjetividad entendida como deseo y esfuerzo y un mundo en el que esa subjetividad se expresa y se objetiva. Con ello trataremos de llevar la atención hacia el punto común de ambas metodologías de la reflexión: el sujeto capaz de sentirse a sí mismo de un modo interrogante y de volver sobre su propia actividad constitutiva del sentido del mundo.

\section{El CONCEPTO METOdOLÓGICO DE REFLEXIÓN EN EDMUND HUSSERL}

El texto de 1913, Ideas relativas a una fenomenología pura y a una filosofía fenomenológica, es fruto del esfuerzo por fundar una nueva filosofía que se pregunta principalmente por la posibilidad del conocimiento. Este trabajo venía tejiéndose desde la publicación de las Investigaciones lógicas, en 1900 y 1901, y en cursos posteriores que Husserl impartía en Göttingen, de los cuales quizá el más conocido es el de 1904/05, del cual salieron las célebres Lecciones sobre la conciencia interna del tiempo, además de otros textos hoy publicados en Husserliana. En 1911 se publica en la revista Logos un extenso artículo de Husserl, titulado La filosofía como ciencia estricta, donde se prepara el terreno de la fenomenología haciendo una crítica a las concepciones de la ciencia y la filosofía vigentes, principalmente una crítica del psicologismo, del que Husserl quería distanciarse. Antes, en 1907, Husserl había dado un curso que se publicaría como La idea de la fenomenología, en el que de igual modo se daba una introducción a la futura filosofía.

Todos estos trabajos convergen en el texto de 1913, Ideas relativas a una fenomenología pura y a una filosofía fenomenológica, que constituye un manual 18.

${ }^{1}$ Cfr. Edmund Husserl, Meditaciones cartesianas, Madrid, Fondo de Cultura Económica, 2009², § 
de estudio de fenomenología. El concepto que nos interesa se entreteje en este trabajo con el de la variación eidética y, en mayor medida, con el de la reducción trascendental. Está en juego la gran operación y la gran actividad filosófica en que consiste la fenomenología. Nosotros, como hemos dicho, vamos a detenernos sólo en el concepto de reflexión en tanto que concepto metodológico, para ver después su recuperación por parte de Ricoeur. En el parágrafo $§ 77$ tenemos una importante indicación a propósito de la reflexión:

Entre las más universales peculiaridades esenciales de la esfera para las vivencias tratamos en primer lugar la reflexión. Lo hacemos así en gracia a su universal función metodológica; el método fenomenológico se mueve íntegramente en actos de la reflexión. ${ }^{2}$

¿Por qué este valor metodológico de la reflexión? Diremos que, de modo general, la actividad de la reflexión se caracteriza por la capacidad de desplazar la atención, en una vivencia, del objeto vivido a la forma en que se vive ${ }^{3}$. De modo paradigmático, en el caso de la percepción externa la reflexión permite pasar de un modo de atención en el que la conciencia está volcada sobre el color azul de un cuadro, para atender ahora al modo de vivencia que podemos denominar con la expresión "percibir el color azul". Esta posibilidad tiene como origen, y también como finalidad, la pregunta, "¿qué es en general percibir un color?". Husserl nos dirá que este tipo de reflexión vuelta sobre la percepción externa es el más sencillo de llevar a cabo, mientras que, por el contrario, la reflexión sobre los actos anímicos será mucho más difícil, sino imposible. Tanto en el ejemplo de la alegría como en el ejemplo de la cólera, es difícil que la reflexión corra paralela al acto mismo. Es decir, en la alegría o en la cólera es difícil reflexionar sin modificar la disposición afectiva en la que me encuentro. En general, la reflexión sólo puede aparecer cuando la alegría o la cólera empiezan a disiparse. En cambio, en el caso de la contemplación del cuadro, percepción y reflexión pueden correr casi paralelas y ser contemporáneas. En todo caso, definiremos la reflexión como aquella actividad de la conciencia capaz de volverse sobre el resto de actividades, sea para examinar la idealidad de los

\footnotetext{
${ }^{2}$ Edmund Husserl, Ideas relativas a una fenomenología pura y a una filosofía fenomenológica I, Madrid, Fondo de Cultura Económica, 1993, § 77, p. 172 (en adelante, Ideas I). En traducción de Antonio Zirión, que está en proceso de ser publicada: "Entre las más generales peculiaridades esenciales de la esfera pura de las vivencias tratamos en primer lugar la REFLEXION. Lo hacemos así en gracia a su GENERAL función metodológica: el método fenomenológico se mueve íntegramente en actos de reflexión".

${ }^{3} \mathrm{Cfr}$. Meditaciones cartesianas, § 15.
} 
objetos sobre los que estas actividades están vueltas, sea para acceder a la esencia misma de estas actividades.

La fenomenología, en tanto doctrina de la esencia de las vivencias ${ }^{4}$, necesita servirse de la reflexión como su instrumento. Ahora bien, ¿diremos con ello que la reflexión es -tan sólo- una bisagra capaz de hacer o dejar girar la atención de la conciencia hacia sí misma? En principio, la reflexión podría llevar a cabo este giro de la atención, de la "mirada", desde cualquier objeto vivido por la conciencia hacia la forma o el modo propio en que ese objeto aparece y se da. Así, habría también una reflexión en el recuerdo, una reflexión en la fantasía, etc. Con ello, como se ha sugerido ya, tendríamos la posibilidad de principio de acceder a la esencia de tales actividades, lo que nos permitiría, por ejemplo, poder establecer una diferencia entre la esencia de la percepción, de la memoria y de la fantasía -por poner un ejemplo de actos clásicos de la fenomenología.

Cabe preguntar ahora: ¿se da con ello por supuesta y conocida la esencia de la reflexión? ¿Es necesaria una reflexión sobre la reflexión o hay que entender que, antes de que la reflexión se vuelva sobre cualquier otra actividad, la reflexión ya se ha vuelto sobre sí misma y ya ha comprendido su esencia? En último término no habría que decir que la reflexión se dedica a extraer la esencia de cada acto particular, para luego hacer una suma y una comparativa de todos los actos reflexionados. Más que eso, la reflexión puede atender también a los elementos concretos de cada acto y, más aún, a las relaciones universales que ligan unos actos con otros en la corriente de conciencia.

La reflexión es, según lo acabado de exponer, un rótulo que designa actos en que resulta apresable con evidencia y analizable la corriente de las vivencias con todo cuanto de variado sobreviene en ella (componentes de vivencias, correlatos intencionales). La reflexión es, así podemos decirlo también, el rótulo que designa el método de conciencia para el conocimiento de la conciencia en general, pero justo en este método se convierte ella misma en objeto de posibles estudios: la reflexión es también el rótulo de vivencias esencialmente relacionadas, o sea, el tema de un importante capítulo de la fenomenología. Distinguir las diversas "reflexiones" y analizarlas íntegramente en orden sistemático, es su tema. ${ }^{5}$

Habría que preguntar ahora, supuesta ya la posibilidad de este giro de la atención hacia de lo vivido a la vivencia, ¿de qué modo tiene que mirar la re-

\footnotetext{
${ }^{4} \mathrm{Cfr}$. Edmund Husserl, Ideas I, § 71.

${ }^{5}$ Ibidem, § 78, p. 176.
} 
flexión a la vivencia para poder extraer de ella su esencia? No resulta difícil, según el ejemplo de Husserl, que miremos una hoja de papel y tratemos de llevar la atención no hacia la hoja como tal, sino en tanto que hoja percibida por mí, entendida como hoja de color blanco que yo percibo. De alguna manera accedemos con facilidad a la intuición de la percepción del color. Pero, por otro lado, una intuición de este tipo se nos escapa, no por su supuesta complejidad, sino sencillamente por su obviedad. En efecto, descrita como hasta ahora, la reflexión en concreto, y la fenomenología en general, podrían no servir a otra cosa que a darnos a conocer un inventario de obviedades -como la percepción del color, la percepción del sonido, etc. - si no fuera porque algunas de estas obviedades, examinadas con la lupa fenomenológica, pueden llegar a despertar el sentimiento de asombro con el que hemos empezado esta breve investigación. La cuestión delicada se plantea en el momento en que queremos entender que la reflexión sobre la reflexión no sólo nos da acceso a la esencia de un acto particular, sino que nos permite entrever también el sentido de una actividad muy especial del ser humano, que podríamos denominar conciencia de la conciencia o segunda conciencia.

Husserl nos dirá, según la cita anterior y de modo preliminar, que hay un capítulo de la fenomenología dedicado a la reflexión sobre la reflexión o a la reflexión sobre las diferentes "reflexiones" posibles. Como hemos dicho, este capítulo no debería de tener por mera finalidad la de hacer un inventario que permitiese contabilizar el número de "reflexiones" posibles, enumerar sus semejanzas y sus diferencias, etc. Habría que preguntar si el todo vale más que la suma de sus partes o, de un modo más claro, si el sentido de la actividad reflexiva sobrepasa el estudio de los diferentes actos reflexivos concretos, vueltos sobre percepciones, juicios, etc. Para el caso que nos ocupa, habría que ver si una suerte de reflexividad inmanente entreteje las diferentes reflexiones, dando un sentido unitario al rótulo general "reflexión", comprendido a su vez a una con los rótulos generales "percepción", "memoria", "fantasía" y, en último término, dentro del rótulo general "conciencia" o "vida intencional", pues, en último término, de lo que se trata es de conocer la subjetividad trascendental que se da a sí misma el mundo, incluyéndose como vida que vive en el mundo ${ }^{6}$.

${ }^{6}$ Cfr. Ibidem, §§ 33 y 34. 
Toda vivencia es en sí misma un río de generación, es lo que es una creación radical de un tipo esencial invariable: un río constante de retenciones y protenciones unificado por un aspecto, él mismo fluyente, de originariedad, en que se tiene conciencia del vivo ahora de la vivencia frente a su "antes" y "después". Por otra parte, tiene toda vivencia sus paralelos en diversas formas de reproducción que podemos considerar como transformaciones "operativas" ideales de la vivencia primitiva; toda vivencia tiene su vivencia "exactamente correspondiente", y, sin embargo, de una modalidad completamente distinta, en un recuerdo, así como en una posible expectativa, en una posible mera fantasía y también en las reiteraciones de estas variaciones. ${ }^{7}$

Como hemos sugerido, hace falta que la reflexión se supere a sí misma como un acto concreto que se vuelve sobre una vivencia particular; por exceso y por defecto. Por un lado, hace falta que en su trabajo metódico la reflexión encadene sus diferentes "miradas" a las vivencias de un modo que sea más que el de la suma de los diferentes resultados extraídos por sus operaciones. Por otro lado, es necesario que subyazca a la reflexión un tipo de reflexividad inmanente, que sería la que permite dar una continuidad de fondo a la alternancia entre las vivencias y sus reflexiones. En general, esta reflexividad inmanente está implicada en la misma corriente de conciencia como tal. En primer lugar, hay actos de dirección inmanente en los cuales la propia vivencia está ya vuelta sobre la subjetividad, antes de toda reflexión: actos de dirección inmanente donde la vivencia coincide con lo vivido o -dicho de otro modo-donde el objeto intencional pertenece a la propia corriente de la conciencia. Husserl hablará a este propósito de actos de percepción inmanente ${ }^{8}$. En segundo lugar, como veremos, también los actos de dirección trascendente tienen la peculiaridad de principio de estar prestos a ser percibidos por la reflexión ${ }^{9}$. Esto sería fruto de una particularidad de la corriente de las vivencias en tanto corriente temporal ${ }^{10}$. Una segunda intencionalidad, no desligada de la intencionalidad volcada sobre los objetos del mundo, estaría vuelta sobre sí misma de modo constante y continuo, modulando este carácter de estar toda vivencia presta para ser percibida por la mirada de la reflexión.

Por un lado, pues, la reflexión nos conduce a la temporalidad inmanente por vía de la retención. Por el otro lado, la "mirada", capaz de encadenar todas las reflexiones concretas en una sola contemplación unificadora, da lugar a la

\footnotetext{
${ }^{7}$ Ibidem, § 78, p. 177.

${ }^{8} \mathrm{Cfr}$. Ibidem, § 38.

${ }^{9} \mathrm{Cfr}$. Ibidem, § 45.

10 Véase, al respecto, Edmund Husserl, Lecciones de fenomenología de la conciencia interna del tiempo, Madrid, Trotta, 2002; especialmente la sección tercera y los apéndices.
} 
idea o a la imagen del espectador fenomenológico. Este espectador reflexivo es un espectador de sí, cuya reflexión no consiste en ponerse a sí mismo como sujeto de las acciones, sino como observador desinteresado de la actividad del yo en tanto subjetividad trascendental. Con ello hay que tener presente que el espectador fenomenológico no lleva a cabo una reflexión natural, sino trascendental, y que el "observado", aunque es el yo en actitud natural, "interesado" en cierto modo - pero sobre todo olvidado de sí en la actividad intencional-, es contemplado desde la perspectiva de su subjetividad trascendental. Dicho de otro modo, la reflexión no es reflexión natural ni psicológica y el reflexionado no es el sujeto en actitud natural, el sujeto psicofísico. Tanto es así que la reflexión fenomenológica puede interesarse por una reflexión natural, con el fin de descubrir y describir la esencia de la así llamada "reflexión natural" y sus posibilidades generales. Ahora -Husserl llevará al extremo estos ejemplos en Filosofía primera-, al llevar a cabo el espectador fenomenológico una reflexión sobre el sujeto olvidado de sí en la actitud natural, el mismo espectador fenomenológico no se toma a sí mismo por objeto, y necesitaría a su vez un nuevo espectador capaz de poner su propia actitud como "tema". Con ello se abre la posibilidad de despliegue infinito de miradas, lo que, antes de envolvernos en un circulo absurdo, permite presentir un sentido último de la actividad fenomenológica.

Podría decirse, con cierta justicia, que sería necesario poner a distancia toda la obra de Husserl para intuir lo mejor posible la esencia y el sentido de la gran actividad reductiva y reflexiva en que consiste la fenomenología. De otro lado, habría que penetrar en la temporalidad inmanente, impregnada de componentes afectivos y emotivos, para vislumbrar la esencia y el sentido del serpara-sí de la conciencia, aquel que, sin coincidir exactamente con la actividad reflexiva del espectador fenomenológico, tampoco se reduciría a ser una suerte de actividad reflexiva pasiva, espontánea y, en todo caso, circunscrita a la actitud natural. Si es posible, como hemos sugerido, separar al espectador fenomenológico del sujeto trascendental, habría que evaluar la posibilidad de diferenciar la esencia de dos actividades: una, la actividad reflexiva del propio espectador, propiamente fenomenológica en sentido metódico; otra, la actividad reflexiva del sujeto trascendental, desembarazada del compromiso metodológico sin ser sin embargo una reflexión puramente natural volcada sobre actos concretos. De otro modo, sería cuestión de interrogar una posible diferencia 
entre el ser-para-sí del espectador desinteresado y del ser-para-sí del sujeto trascendental como tal, anterior a la escisión que implican los actos reflexivos particulares. Ya hemos sugerido la vía de investigación de la temporalidad inmanente, que ahora sin embargo no podemos emprender.

\section{El CONCEPTO METOdológico de REFLEXión en PAUl Ricoeur}

El filósofo francés Paul Ricoeur es conocido hoy en día como hermeneuta; seguramente como el más importante de la lengua francesa. Es importante señalar sin embargo que entre las muchas influencias que ayudaron a forjar su filosofía, fue la fenomenología de Husserl una de las más importantes. Cabe recordar que Paul Ricoeur fue el traductor al francés del primer volumen de las Ideas relativas a una fenomenología pura y a una filosofía fenomenológica, que se publicaría en 1950, el mismo año en que se publica su tesis doctoral, Lo voluntario y lo involuntario. Gran parte de la traducción la hizo Ricoeur durante su cautiverio en la segunda guerra mundial, lo que le da un valor simbólico añadido a su valor histórico de introducción a la fenomenología en Francia.

En lo que respecta al concepto de reflexión, Ricoeur conjuga la fenomenología, entendida en el contexto alemán que llegaría hasta Kant pasando por Fichte, con la tradición francesa que parte de Descartes y pasa por Maine de Biran y Jean Nabert. Desde este conglomerado de influencias, Ricoeur hace suyo el problema del acceso a la subjetividad a través de la reflexión o, dicho de otro modo, de la aparición y aprehensión de la conciencia de sí al interior de la conciencia intencional. Ya en Lo voluntario y lo involuntario Ricoeur entiende que el yo no puede reflexionar si no es a partir de sus actos. Ahora bien, Ricoeur llevará esta exigencia más allá de Husserl, poniendo cada vez más el acento en el lado noemático de la correlación intencional y pasando de los actos a las obras.

La elaboración metodológica del concepto de reflexión, que señala este camino de los actos a las obras $-y$ de la fenomenología a la hermenéutica-, se forja sobre todo en los años 60, exponiéndose primero en De l'interpretación. Essai sur Freud, publicado en 1965 y traducido en español como Freud: una interpretación de la cultura, y después en Le conflit des interpretations, de 1969. En el primero de los textos, dedicado a Freud - pero también dedicado a 
la interpretación-, Ricoeur dará ya clara definición de lo que durante muchos años va a entender por "reflexión". Como hemos dicho, su herencia es muy compleja, y abarca por un lado a Husserl, a Fichte y a Kant y, por otro lado, a Nabert, a De Biran y a Descartes, por citar sólo algunos autores. En primer lugar, la lectura de Freud descubre a Ricoeur la necesidad de articular la fenomenología de la conciencia con la interpretación del inconsciente. Dicho de otra manera, más allá de una reflexión donde sea posible descubrir y describir con claridad la esencia de las vivencias, Ricoeur previene de ciertas zonas de la subjetividad donde la conciencia raya con lo inconsciente y donde la descripción deja paso a un trabajo de interpretación.

¿Cómo afecta esto a la reflexión? Su transformación no es para nada leve: pasamos de una reflexión como intuición directa y evidente de vivencias que no dejan lugar a zonas oscuras - argumento que Husserl repetirá varias veces- a una reflexión entendida como interpretación, a reflexión necesitada de interpretación ${ }^{11}$. No se quiere decir con ello que una reflexión sustituya a la otra, sino, más bien, que una envuelve a la otra. La reflexión fenomenológica opera allí donde no hay lugar al equívoco, donde pueden llegar a describirse con claridad y distinción las operaciones con las que la subjetividad trascendental da sentido al mundo. La condición de esta filosofía inequívoca es la reducción trascendental, que tiene como cometido, entre otros, desembarazarse de relativismos culturales o históricos. La reflexión hermenéutica, en cambio, opera allí donde se supera la evidencia intuitiva y emana una significación que puede ser interpretada en diferentes direcciones, teniendo que extraer su comprensión más profunda del conflicto entre las mismas. He aquí una primera definición de este nuevo concepto metodológico de reflexión:

Reflexión no es intuición o, dicho en términos positivos: la reflexión es el esfuerzo por volver a captar el Ego del Ego Cogito en el espejo de sus objetos, de sus obras y finalmente de sus actos. Ahora bien, ¿por qué la posición del Ego debe volver a ser captada a través de sus actos? Precisamente porque no está dada ni en una evidencia psicológica ni en una intuición intelectual ni en una visión mística. Una filosofía reflexiva es lo contrario de una filosofía de lo inmediato. ${ }^{12}$

\footnotetext{
${ }^{11}$ Paul Ricoeur, Le conflit des interprétations. Essais d'hermenéutique, Paris, Seuil, 2011, pp. 1921.

12 Paul Ricoeur, Freud, una interpretación de la cultura, México, Fondo de Cultura Económica, 2007, p. 41. Véase también Le conflit des interprétations, pp. 321-329.
} 
Para Ricoeur, el ego está olvidado de sí en los objetos en los que se vuelca intencionalmente. Dicho de otro modo, el cogito cogitatum hace desaparecer al ego, que queda puesto entre paréntesis. Para reapropiarlo es preciso seguir su huella, aquella que éste ha dejado en el mundo. No sirve entonces una vuelta sobre sí, sino todo un rodeo por el mundo, principalmente por el mundo del lenguaje en el que el ego se expresa. La reflexión no consiste en este caso en un giro sobre la subjetividad y sus actividades intencionales, sino en una atención a las obras que condensan esas actividades y que forman parte de la cultura y de la historia. Ahora bien, ¿qué es lo que trata de reapropiarse esta reflexión? ¿En qué modo podemos decir que la reflexión conserva todavía el rasgo más general de un giro de la mirada? Aquí Ricoeur no opta por una vuelta sobre la subjetividad en tanto actividad constitutiva del mundo y fundamento del conocimiento, sino por una vuelta a la actividad más práctica, implícita en la intencionalidad entendida como acción y emparentada más con una ética que con una filosofía entendida como fundamento del conocimiento.

\begin{abstract}
La reflexión es ética antes de convertirse en una crítica de la moralidad. Su fin es captar el Ego en su esfuerzo por existir, en su deseo de ser. Una filosofía reflexiva encuentra y quizá salva aquí la idea platónica de que la fuente del conocimiento es en sí misma Eros, deseo, amor, y la idea spinoziana de que es conatus, esfuerzo. Este esfuerzo es un deseo, porque jamás se satisface, pero este deseo es un esfuerzo, porque es la posición afirmativa de un ser singular y no simplemente una falta de ser. Esfuerzo y deseo son las dos caras de la posición del Sí en la primera verdad: yo soy. ${ }^{13}$
\end{abstract}

No es difícil apreciar que en esta reivindicación de una reflexión hermenéutica Ricoeur se aleja de la fenomenología tanto como se acerca a una ontología. Esfuerzo y deseo, en un fuerte sentido, pasan a ocupar la fuente de la que han de brotar los actos de los cuales la reflexión ha de dar cuenta a la hora de comprender la subjetividad. Con ello se deja de lado, por ejemplo, una fenomenología de la percepción, de la memoria o de la imaginación, entendidas estas dimensiones de la vida intencional como constituyentes del espesor y de la profundidad del horizonte de mundo vivido ${ }^{14}$. La reflexión, entonces, ya no trata de describir la constitución del mundo, sino de comprender las expresiones de una vida. Para ello, una gran diferencia se juega en el modo de llevar la atención bien hacia el lado noético bien hacia el lado noemático de la correlación vida-

13 Paul Ricoeur, Freud, una interpretación de la cultura, p. 43.

${ }^{14}$ Ricoeur ya había optado, en Lo voluntario y lo involuntario, por un tipo de fenomenología, que, sin oponerse directamente a la Fenomenología de la percepción de Merleau-Ponty, diera más valor a la dimensión práctica que a la teórica y, con ello, más valor a la voluntad que a la percepción. 
mundo. Una fenomenología del deseo y del esfuerzo sigue siendo posible; y posible en el modo en el que se ha descrito, es decir, mediante reflexiones vueltas sobre actos concretos de "desear" o de "esforzarse". Ahora bien, en Ricoeur es preciso que haya una expresión del deseo para que la reflexión sea posible: "La reflexión es la apropiación de nuestro esfuerzo por existir y de nuestro deseo de ser, a través de las obras que atestiguan ese esfuerzo y ese deseo" ${ }^{15}$.

El problema que se pone es el de la necesidad de que la reflexión se realice a través y sólo a través de las obras en que se expresa el deseo y el esfuerzo. Para Ricoeur el acto no llega a significar el deseo de modo suficiente; es preciso ver el lugar a donde el acto apunta, y no sólo, sino y sobre todo tratar de comprender aquello que el acto realiza, su marca, su huella, aquella que hay que interpretar. Según este modelo, todo eidos del deseo no podría ser despejado sino es a partir de su telos constituido, el cual quedaría no obstante en un margen de ambigüedad ${ }^{16}$. Aquí las diferencias son importantes entre una y otra metodología reflexiva. En primera instancia, Ricoeur ve imposible una intuición eidética del deseo y del esfuerzo en los actos en los que estas dimensiones de la subjetividad podrían expresarse. El rodeo por la obra es para Ricoeur insoslayable. En segundo lugar, este principio de la metodología hermenéutica abandona en cierto modo el recurso a la reducción fenomenológica y en su lugar invita a la así llamada "vía larga", que Ricoeur expondrá en El conflicto de las interpretaciones: "La reflexión debe convertirse en interpretación, porque no puedo captar este acto de existir más que en signos dispersos en el mundo"17.

Lo que está aquí implicado es la necesidad de reconocer que toda vuelta sobre sí -el vínculo que aún mantienen ambos conceptos de reflexión- pasa por el lenguaje, y que todo lenguaje en el que el sí se expresa y se reconoce es un lenguaje equívoco, donde un sentido literal esconde un segundo sentido. Así las cosas, la reflexión debe convertirse en interpretación. Dicho de otro modo,

${ }^{15}$ Paul Ricoeur, Freud, una interpretación de la cultura, p. 44. Ver también Le conflit des interprétations, pp. 21-25

${ }_{16}$ Otro momento de difícil armonía entre fenomenología y hermenéutica aparecería aquí, al afirmar Ricoeur que "la posición de ese esfuerzo o ese deseo no solamente está privada de toda intuición, sino que no está atestiguada más que por obras cuya significación queda dudosa y revocable" (Freud, una interpretación de la cultura, p. 44). Queda desestimado aquí, entonces, el recurso a una evidencia intuitiva sobre la esencia del deseo y el esfuerzo humanos, anulándose con ello el conocido principio de todos los principios que Husserl expone en el $\S 24$ de Ideas $I$, al menos en lo que se refiere a la posición e intuición del deseo.

${ }_{17}$ Paul Ricoeur, Freud, una interpretación de la cultura, p. 44. 
la reflexión será sólo una etapa que ha de ser superada por la interpretación dentro del método hermenéutico. Este método abandona definitivamente el nivel de la subjetividad trascendental para partir de la existencia, atravesada por alteridad histórica y cultural y medida en todo momento por el lenguaje. Corresponde a este método un largo rodeo - Ricoeur lo llamará vía larga - cuyo fin no es de todos modos una vuelta a la fenomenología trascendental, sino a una ontología del deseo y el esfuerzo. Éste es un camino que aquí sólo podemos señalar, al igual que sólo podemos mencionar, en el caso de Husserl, la necesidad de comprender la reflexión en el contexto de la reducción fenomenológica, sin poder en este trabajo completar estas exigencias. Nos quedaremos ahora expresamente con la relación entre Husserl y Ricoeur, sobre todo a propósito de la reflexión.

\begin{abstract}
[Husserl] concibe la fenomenología no sólo como un método de descripción esencial de las articulaciones fundamentales de la experiencia (perceptiva, imaginativa, intelectiva, volitiva, axiológica, etcétera), sino como una autofundamentación radical en la más completa claridad intelectual [...]. Sin embargo, la fenomenología, en su ejercicio concreto y no en la teorización que aplica a sí misma y a sus pretensiones últimas, señala ya el alejamiento en lugar de la realización, del sueño de esa fundamentación radical en la transparencia del sujeto con respecto a sí mismo. El gran descubrimiento de la fenomenología, sometida al requisito de la reducción fenomenológica, es la intencionalidad, es decir, en su sentido menos técnico, la supremacía de la conciencia de algo sobre la conciencia de sí. Pero esta definición de la intencionalidad es aún trivial. En su sentido riguroso, la intencionalidad significa que el acto de referirse a algo sólo se logra a través de la unidad identificable y reidentificable del sentido referido -lo que Husserl llama el noema, o correlato intencional de la referencia noética. Además, sobre este noema se deposita en estratos superpuestos el resultado de las actividades sintéticas que Husserl denomina constitución. Ahora bien, la tarea concreta de la fenomenología -en particular en los estudios dedicados a la constitución de la cosa- pone de manifiesto, de modo regresivo, estratos cada vez más fundamentales donde las síntesis activas remiten continuamente a síntesis pasivas cada vez más radicales. La fenomenología queda así atrapada en un movimiento infinito de interrogación hacia atrás en el que se desvanece su proyecto de autofundamentación radical. Incluso los últimos trabajos consagrados al mundo de la vida designan con este término un horizonte de inmediatez que nunca se alcanza. El Lebenswelt nunca está dado y siempre se presupone. Es el paraíso perdido de la fenomenología. ${ }^{18}$
\end{abstract}

Si he querido transcribir esta extensa cita, es porque aquí está en juego una gran tensión entre fenomenología y hermenéutica, que exige tomar un camino u otro. Para este caso, parece no ser posible una vía intermedia o, según la metodología del propio Ricoeur, una vía que extraiga sus conclusiones de la

${ }^{18}$ Paul Ricoeur, Del texto a la acción. Ensayos de hermenéutica II, Buenos Aires, Fondo de Cultura Económica, 2000, p. 29.

| Investigaciones Fenomenológicas, n. 9, 2012. 
tensión entre ambas metodologías. En el texto de 1965, Ricoeur enunciaba la posibilidad de conjugar lo que él llamaba la arqueología del sujeto de Freud con la teleología de la conciencia de Hegel. El presupuesto era precisamente el de la dependencia de ambas perspectivas. Ahora bien, no podemos decir lo mismo del caso de la fenomenología, pues Ricoeur no entiende que deba ser arbitrada con otra filosofía; para él, la fenomenología debe ser superada por la hermenéutica. La reflexión, como hemos visto, debe convertirse en interpretación. Hay que decir, sin embargo, que no es imposible pensar que el trabajo infinito de explicitación del sujeto trascendental pueda ir de la mano de un trabajo igualmente infinito- de descifrado de las obras y los textos en los que ese mismo sujeto se expresa. Ricoeur, después de haber incluido a Husserl en las pretendidas filosofías de la transparencia absoluta, no ve en el anuncio de la fenomenología como tarea infinita sino un fracaso; fracaso que él no toma en consideración como posibilidad. La fenomenología genética que se enuncia al trasluz de sus referencias a las síntesis pasivas queda así desestimada.

Ya lo hemos dicho varias veces. Ricoeur pone todo el acento de su investigación hacia el lado noemático de la correlación vida-mundo, en una filosofía del rodeo que va de los actos a las obras, para volver sólo al final hacia una subjetividad entendida principalmente como deseo de ser y esfuerzo por existir. Con ello entiende que Husserl se ha quedado atrapado en el lado noético, en una regresión in infinitum que escarba en las capas constituyentes más pasivas de la subjetividad, justo en aquellas en las que se forman las primeras unidades de sentido. A mi juicio, la insistencia de Ricoeur en que su método volcado sobre el lado noemático debe volver al lado noético no es menor que la insistencia inversa, en Husserl, de profundizar el lado noético con el fin de llegar al lado noemático. En efecto, Husserl menciona la posibilidad de elaborar una filosofía del arte o de la historia y, sobre todo, afirma que la fenomenología no es el trabajo de una sola persona, sino de generaciones de investigadores. Esta idea, que seguramente concuerda en valor con la idea que Ricoeur tenía de su método hermenéutico, nos lleva a contrastar la fenomenología y la hermenéutica de un modo mucho menos dicotómico.

A mi juicio, es motivo común de ambas filosofías una investigación infinita, pues Ricoeur entiende en varios lugares que el largo rodeo por el lenguaje, si bien tiene que volver siempre sobre el sujeto que se pone como existente, nunca termina en un final absoluto - de otro modo se condenaría a sí mismo, te- 
niendo para su filosofía un deseo de transparencia y de inmediatez mayor que el de las filosofías que ajusticia. En este sentido, resulta extraño que el filósofo de la mediación entre unas y otras tradiciones filosóficas haya cerrado la puerta a la fenomenología genética, aquella que precisamente había sobrepasado la idea de una inmediatez absoluta del cogito consigo mismo en el programa de una investigación infinita, impidiendo así ver una mayor y mejor armonía entre el planteamiento que él propone de la reflexión $-y$ de la hermenéutica en general- y el de la reflexión fenomenológica.

\section{BREVE ESBOZO DE UNA GENÉTICA DE LA REFLEXIÓN}

La vía larga que propone Ricoeur se aleja de todo presentimiento y sentimiento inmediato de sí, llevando la comprensión a dimensiones cada vez más mediatizadas por el lenguaje. El caso más paradigmático es la formulación de la identidad narrativa, esto es, de una comprensión de sí articulada por el ejercicio de un narrarse a sí mismo según formas y figuras extraídas de las narraciones que heredamos. Este alejamiento de la vía regresiva es también un abandono de la pregunta por las condiciones mismas de la reflexión, olvidadas en la explicitación metodológica de la hermenéutica y en su aplicación como interpretación. Se abandona así el camino de lo que podríamos llamar en fenomenología una genética de la reflexión, en el que Ricoeur mismo parecía querer adentrarse en sus primeros trabajos:

Debe haber una referencia a sí mismo que no es todavía una mirada sobre sí, sino cierta manera de relacionarse o de comportarse con relación a sí mismo, una manera no especulativa, o mejor no espectacular: una implicación de sí mismo rigurosamente contemporánea del acto de decisión y que es, de alguna manera, un acto respecto de sí mismo. Esta implicación de sí mismo es la que debe tener en germen la posibilidad de la reflexión. ${ }^{19}$

Como sabemos, Ricoeur opta por no seguir esta vía o por posponerla hasta después de su largo rodeo por los documentos en los que se expresaría esta suerte de referencia a sí no explicitada por la reflexión. Pues bien, aquí se quiere proponer la vía de una fenomenología genética, vuelta sobre las dimensiones

${ }^{19}$ Paul Ricoeur, El proyecto y la motivación. Lo voluntario y lo involuntario I, Buenos Aires, Docencia, 1986, pp. 71s. 
constituyentes y constitutivas de la reflexión, aquellas que hacen posible precisamente el giro de la mirada, que es el punto común que mantendrán las descripciones de Husserl y de Ricoeur ${ }^{20}$. Una genética de la reflexión, como ya hemos apuntado, tendría que investigar la constitución de la temporalidad inmanente de la conciencia, en busca de este tipo de intencionalidad longitudinal o segunda que permite la referencia a sí. Habría que ver también el modo en que la esfera del sentimiento es una esfera de doble intencionalidad, en donde cabría un sentirse o un presentirse previo a la vuelta reflexiva sobre sí. Ello incluiría, en cierto modo, una investigación sobre el deseo y sobre el esfuerzo que Ricoeur propone como principios de la acción humana. En los términos infinitivos en los que la fenomenología pone la cuestión, hablaríamos de una genética del desear y de una genética del esforzarse, lo que nos llevaría a investigar la raíz del esfuerzo - como hace en alguna medida la fenomenología de Michel Henry- y no sus frutos.

A mi juicio, esta vía - de dirección prohibida en la hermenéutica de Ricoeur- no está privada de intuitividad. Sin entrar en dicotomías, y con el ánimo de la mediación que propone el concepto de dialéctica en Ricoeur, creo posible y pertinente hacer el camino de una fenomenología genética que tuviese como objetivo la explicitación de la intuitividad del desear, con el fin de articularla con una hermenéutica del sí mismo en que examinase, por ejemplo, la capacidad del giro de la mirada a partir de las narraciones autobiográficas. De alguna manera, una genética trataría de asistir al nacimiento del narrador hermenéutico y también del espectador fenomenológico; no para alcanzar una inmediatez y transparencia absolutas, sino todo lo contrario, para entrever el "lugar" en el que se produce la constante brecha del sujeto consigo mismo, para ganar intuitividad en aquellas capas de la vida pasiva en las que brota una alteridad y alteración inmanente.

\section{CONCLUSIONES}

A la forma de ser de la vivencia es inherente que a toda vivencia real, viva como presencia originaria, pueda dirigirse en la forma más directa una mirada de percepción in-

\footnotetext{
20 "La reflexión es el acto de volverse sobre sí por el cual un sujeto vuelve a captar, en la claridad intelectual y la responsabilidad moral, el principio unificador de las operaciones en las que se dispersa y se olvida como sujeto". Paul Ricoeur, Del texto a la acción, p. 28.
} 
tuyente. Esto sucede en la forma de la "reflexión", que tiene la notable propiedad de que lo aprehendido perceptiblemente en ella se caracteriza por principio como algo que no sólo existe y dura dentro de la mirada percipiente, sino que ya existía antes de que esta mirada se volviese a ella. "Todas las vivencias son conscientes" quiere decir, pues, que no son sólo conciencia de algo, ni existen como tal conciencia, sólo cuando ellas son objetos de una conciencia refleja, sino que ya antes de toda reflexión están ahí como "fondo" y por ende en principio prestas a ser percibidas. ${ }^{21}$

A propósito del carácter de las vivencias, Ricoeur podría decir que, además de estar prestas a ser percibidas, las vivencias piden ser expresadas. Su camino es el del rodeo por la expresión, mientras que el de una fenomenología genética sería el de la explicitación de las condiciones trascendentales de una tal prestitud, la que hemos llamado con anterioridad reflexividad inmanente.

Ricoeur sólo ve posible la inteligencia de la vida antepredicativa a partir de la vida predicativa, de igual modo que sólo ve posible una comprensión de lo involuntario a partir de lo voluntario. Con ello, la hermenéutica no es nunca una profundización hacia el interior, sino siempre una búsqueda e interpretación de signos y huellas en el exterior, allí por donde ha pasado el ser humano. Toda vuelta sobre el deseo o el esfuerzo mediante una reflexión directa es para él inútil. Pues bien, a mi juicio esta posibilidad no es inútil del todo, sobre todo si se piensa en la expresión que ha de darse de la misma -también a partir de un lenguaje-, pero a partir de un interés expreso de significar el desear desde sí mismo, anterior a toda reflexión de carácter segundo y de toda interpretación. El trabajo de una descripción directa del esfuerzo, no entendido como un trabajo de transparencia e inmediatez absolutas, puede dar algunos frutos que no han sido atendidos por la hermenéutica de Ricoeur. Al tratar de comprender el deseo humano en las huellas que otros seres humanos han dejado a través de diferentes trazos lingüísticos, lo que pretende es vencer una distancia abierta de antemano. Por el otro lado, e insistiendo en una fenomenología que no busca la transparencia absoluta, habría que decir que es posible una fenomenología -que Husserl ha llamado genética- que buscaría precisamente el nacimiento de una tal distancia, alteración interna y entrada afectiva de la alteridad.

En Husserl, la reflexión tenía como cometido atender a las vivencias como tales para descubrir o desvelar su esencia, dando lugar en último término a un conocimiento de la subjetividad constituyente de mundo; en Ricoeur la idea

${ }^{21}$ Edmund Husserl, Ideas I, p. 103. 
estaba puesta en un tipo de subjetividad más histórico y, más que en su conocimiento constituyente del mundo, en su comprensión en tanto constituido por el mundo, entendido como alteridad. El camino de Ricoeur, al incorporar un mayor número de dimensiones históricas, se elaboraba así como una suerte de contrapunto de la reducción fenomenológica, caracterizada por una puesta entre paréntesis de la trascendencia del mundo como tal. El método de Ricoeur se basa en una vía larga que se pone como tarea recorrer, mediante un tipo de reflexión interpretativa, las diferentes dimensiones en las que el ser humano deja sus marcas y huellas. Así, incluso el sentido de la apercepción de sí debe ser comprendido a partir de una interpretación de los documentos en los cuales la conciencia de la conciencia se dice.

Ciertamente, tengo una apercepción de mí mismo y de mis actos, y esta apercepción es una especie de evidencia ${ }^{22}$. Descartes no puede ser desalojado de esta proposición indiscutible: no puedo dudar de mí mismo sin apercibir que dudo. Pero, ¿qué significa esta apercepción? Una certeza, sin duda, pero una certeza privada de verdad; como bien lo comprendió Malebranche contra Descartes, esta captación inmediata es solamente un sentimiento y no una idea. ${ }^{23}$

Ya lo hemos sugerido: una fenomenología genética de la apercepción de sí no está motivada por el deseo último de llegar a una transparencia e inmediatez absolutas. Antes bien, el sentimiento del que aquí se habla sería en gran medida un presentimiento de sí, que nos llevaría por un camino infinito, aunque para nada estéril, en un tipo de reflexión de tipo emocional -como Husserl analiza en Filosofía primera II- que vaya examinando capas diferentes en las que se forjan tal presentimiento. La temporalidad inmanente es uno de estos "lugares" de investigación, de una riqueza y una preciosidad estupefacientes. Incluso el sueño de una ontología del deseo y del esfuerzo tiene mucho que ganar mediante una genética del desear y del esforzarse que atienda precisamente al momento en que estos impulsos y actividades comienzan a ser presentidos como tales y no tanto reconocidos en sus huellas. Aquí, lo hemos dicho, no queremos contraponer un camino a otro, como el camino mejor al camino peor. Antes bien, se entenderá que una metodología como la de Ricoeur

\footnotetext{
${ }^{22}$ Yo subrayaría el carácter de vivencia de esta apercepción: la apercepción misma es una vivencia que como tal ha de ser reflexionada: Ricoeur sólo lo hará al final de su vía larga, principalmente en Sí mismo como otro. Sin embargo, nos parece que Husserl sigue dando dignidad a una reflexión que no ha de dejar de ser nunca reflexión sobre la propia vivencia tal y como esta se da, y, antes de ser expresada, en el momento mismo de pedir expresión.

${ }_{23}$ Paul Ricoeur, Freud, una interpretación de la cultura, p. 42.
} 
no debe superar el tipo de reflexión que expone la fenomenología para convertirse en interpretación, y con ello en hermenéutica, pero tampoco diremos que una fenomenología genética de la reflexión desmiente de raíz todo hallazgo que una hermenéutica como la de Ricoeur pueda obtener a partir de su metodología.

La fenomenología es ya un lenguaje en el que el deseo y el esfuerzo se expresan. Es un lenguaje de la reflexión que pudiendo ser interpretado no por ello tendría que ser superado. La fenomenología es ya más que un sentimiento de la apercepción: es un trabajo que demuestra que la apercepción es más que un sentimiento: es un continuo de reflexiones encadenadas en la corriente de conciencia, vueltas y entretejidas en toda actividad y pasividad, en todo objeto u horizonte $-y$ ello gracias a la capacidad de la apercepción para desbordarse a sí misma más allá del sentimiento inmediato. Ricoeur lo ha dicho a propósito de su lectura de Husserl: la apodicticidad no significa adecuación. Añadimos que este testimonio no obliga ni conduce únicamente a una hermenéutica, y que entreabre y ofrece el camino a una fenomenología infinita. 